\title{
Automatic Detection and Analysis of Boiler Tube Leakage System
}

\author{
S. Shahul Hamid \\ Associate Professor
}

\author{
D. Najumnissa Jamal \\ Professor \\ Department of Electronics \& Instrumentation, \\ B.S.Abdur Rahman University, Chennai, India
}

\author{
Murshitha Shajahan \\ Asst. Professor (S.G)
}

\begin{abstract}
Detection of boiler tube leakage is a very important factor for power plant functioning, as approximately $60 \%$ of boiler outage is due to tube leakages. The traditional method has many drawbacks in leakage detection. In this study acoustic signal processing methods have been used to detect leaks in pressurized systems of utility and industrial power plants. A lab setup is designed and fabricated which mimics the boiler leakage. Leakage Sound waves are detected by transducers. The signal features are extracted. BPNN algorithm is used to study the datasets. Average specificity of $94 \%$ and sensitivity of $92 \%$ are obtained. Results show that the BPNN is able to detect tube leakages from holes of different diameters and distances efficiently. It emerges that this method of detection makes it promising as a real-time detector, which will progress the automatic detection of boiler tube leakage in boilers.
\end{abstract}

\section{Key words}

Acoustic signals, Boiler tube leakage, BPN, Classification, Feature extraction.

\section{INTRODUTION}

Boiler tube leakage is a major cause of outage and as a consequence power generation loss in thermal power plants is huge. Leakage detection in recovery boilers is important to avoid severe damage of equipment [1]. The walls of the furnace are containing evaporating water with high pressure. Fireside corrosion and thermal stress can cause leakages, implying that water or steam comes in contact with the smelt. Water in the smelt can cause explosion with total destruction of the boiler. In addition to this, shut down of power plants, loss of fuel, maintenance time and cost are additional losses. Hence early and easy detection of boiler tube leakage is important for smooth functioning since approximately $60 \%$ boiler outages are due to tube leaks. Power plant Engineers must remain vigilant about boiler tube leaks so that secondary damages to pressure parts such as water wall tubes, heaters, super heater tubes, re-heater tubes and furnace refractory may be avoided [2].

Boiler tube leaks must be detected very early, otherwise leaking steam may further damage adjacent costly parts due to heavy impact. Maintenance cost to secondary damage from boiler tube leaks is very high and repairing the damage requires several weeks to complete. Due to the substantial costs associated with any forced outage, it is imperative to perform routine inspections. In this manner, conditions with the potential to result in failures can be identified, monitored and addressed before they do result in failures. The quantity of makeup water and hissing sound emitted by leaking steam is detected by human ears at the boiler.

There are a number of systems for detection of Tube leakage available in both conventional boilers and recovery boilers [3, 4]. These systems detect the leakage flow or cracking with acoustic sensors. The leakage of power situation's tubes like water wall tubes, super heater tubes, reheater tubes and economizer tube, the detections are traditional method, filtering method, acoustic spectrum analysis [4,5], and the acoustic catheter with acoustic resonance cavity type detectors [6]. In [7] boiler leakage flows are detected by a mass-balance in a Bayesian network. Here the sensitivity of detection decreases the leakage probability. Xi Sun et al [8] proposed a system based on the boiler characteristics. A dynamic PCA model which is suitable for boiler water/steam leak detection was developed. But it only reduced the false alarms that existed in the conventional systems. These conventional methods may detect a tube leak, if it is big enough and could take as much as 72 hours for detection. There are many instances in which thermal power plants have been allowed to run for extended times with un-detected tube leaks due to confusion with absence of advanced monitoring system. Neural networks [9] and intelligent computing techniques are routinely employed in signal classification systems [10]. Fuzzy sets have attracted the growing attention and interest in modern information technology, production technique, decision making, pattern recognition, diagnostics, data analysis, etc. In recent years, the integration of neural networks and fuzzy logic has given birth to new research into Neuro- fuzzy systems. As a result, those systems can utilize linguistic information from the human expert as well as measured data during modeling. Such applications have been developed for signal processing, automatic control, information retrieval, database management and data classification $[11,12]$.

The purpose of this paper is to present a new idea by fabricating an experimental setup to simulate steam leakage in the boiler tube. The acoustic signal during leakage is acquired and analyzed. This system consists of two stages. The first stage is the feature extraction from acquired acoustic signals and the second stage is the classification of these features. The schematic of the diagnostic method is shown in figure 1.

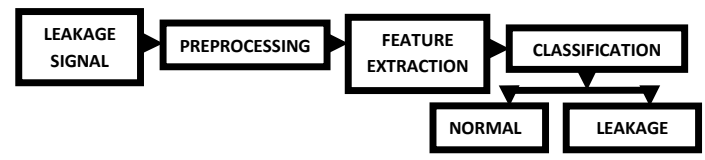

Fig.1. Schematic of the diagnostic method

This paper is organized as follows. Section II describes the experimental setup for data generation. Section III discusses the methodology used for preprocessing and feature extraction from the leakage signals and Section IV deals with the Results and Discussions on how the acoustic signals are analyzed and network model is derived. Conclusions are given in Section V. 


\section{EXPERIMENTAL SETUP}

In order to acquire the acoustic leakage signal, a lab setup model is designed in such a way that the air leakage from the tubes resembles the steam leakage in the boiler tube. For our study we fabricated a boiler tube prototype with 4 long pipes of inner diameter of $8 \mathrm{~mm}$ thickness and $12 \mathrm{~cm}$ length. These pipes will be assumed as a boiler tubes. To assume leakage in boiler a hole for $1 \mathrm{~mm}, 3 \mathrm{~mm}$ holes are drilled. The $1 \mathrm{~mm}$ and $3 \mathrm{~mm}$ holes are coupled with plug. The two $4 \mathrm{~mm}$ holes are coupled with a throttle cum needle valve in order to analyze the leakage under unknown dimension. The needle valve is chosen so that it can bear a pressure up to 300psi. The size of the hole can be varied by throttling the valve. The pressure inlets are provided through which the pressure is given from the compressor. Other two outlets provide in the setup are gate valve and drain valve. The gate valve helps to reduce the pressure immediately. Drain valve helps to remove the condensed water which settles in the tubes.

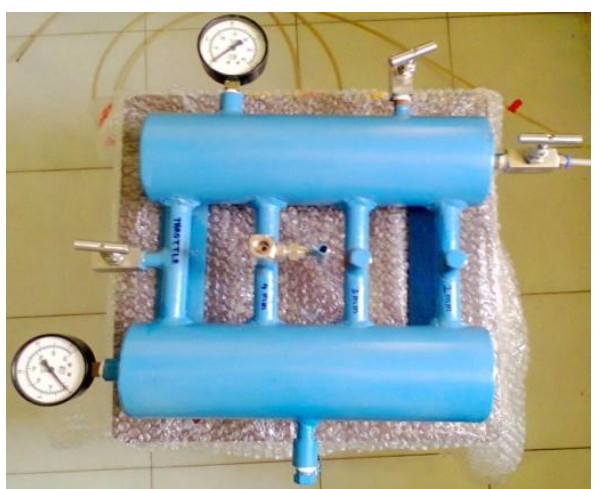

\section{METHODOLOGY}

\subsection{Data recording and preprocessing}

The pressure of about 100psi is applied through the inlet valve of the prototype. Then the plug inserted at $1 \mathrm{~mm}$ hole is removed and the signal is recorded by using the condenser microphone. The recording from the distance of $1 \mathrm{~m}, 2 \mathrm{~m}, 5 \mathrm{~m}$ and 10 meter from the tube leakage system prototype is then preprocessed and used for analysis. In order to find the exact location of the leakage the features are extracted from the signal after filtering the unwanted noise in the signal. A Gaussian filter is used to remove noise and to smoothen the signal

\subsection{Feature extraction}

In order to further reduce the dimensionality of the acquired acoustic signal features are extracted like, Energy, Entropy and some statistical features [10].

\subsubsection{Energy}

It is expressed as

$$
\mathrm{E}=\sum_{\mathrm{i}=1}^{\mathrm{N}} \mathrm{S}_{\mathrm{i}}^{2}
$$

Where $S_{i}=$ signal sample value

The feature energy is taken in order to check the risk level of obtaining the leakage. These features are computed from the acquired leakage signal and they act as input for the classification.

\subsubsection{Entropy}

Recently, [13] have proposed a region based method using ICA bases in which entropy of regions is used as a priority measure. The Shannon entropy function provides a measure of the economy of representation of a signal as given in equation (2)

$$
\text { Entropy }=-\sum p_{i} \log _{2}\left(p_{i}\right)
$$

\subsubsection{Statistical features}

Statistical theory is one of the feature selection method commonly used by researchers. It is a comprehensive analysis method used when several objects and indices are inter-related. The statistical features such as mean and minimum are calculated in this study as one of the feature of the leakage signal.

\subsection{Classification}

The categorization of leakage or background is done by using artificial neural network. Neural networks are being used to analyze leakage signals for the detection of spikes. For identifying the leakage in boiler tubes, the features like Energy, entropy and statistical features like mean and minimum are given as input to ANN. Neural networks are a proven, widely used technology to solve such complex classification problems. In this work, it is proposed to find and use a neural network suitable for identifying boiler tube leakage.

\subsection{Performance}

The performance of the neural networks was estimated using False positive (FP), False Negative (FN), True Positive (TP) and True Negative (TN) values [10]. Classification of a normal data as abnormal is considered as FP and classification of abnormal data as normal is considered FN. TP and TN are the cases where the abnormal is classified as abnormal and normal classified as normal respectively. The performance of the neural networks like accuracy, sensitivity and specificity were estimated using the following relations shown in equations (3), (4) and (5):

$$
\begin{aligned}
& \text { Accuracy }=(\mathrm{TP}+\mathrm{TN}) /(\mathrm{TP}+\mathrm{FP}+\mathrm{TN}+\mathrm{FN}) \\
& \text { Sensitivity }=\mathrm{TP} /(\mathrm{TP}+\mathrm{FN}) \\
& \text { Specificity }=\mathrm{TN} /(\mathrm{TN}+\mathrm{FP})
\end{aligned}
$$

Accuracy is the representation of classifier performance in global sense. Sensitivity and specificity are the proportions of abnormal data classified as abnormal, normal data classified as normal respectively.

\section{RESULTS AND DISCUSSION}

The best parametric representation of acoustic signals is an important task to produce a better recognition performance [14]. Software is written to record the signal for 5 seconds at a sampling rate of $8000 \mathrm{~Hz}$. The recorded signal for $1 \mathrm{~mm}-1$ meter, $3 \mathrm{~mm}-1$ meter, $4 \mathrm{~mm}-1$ meter and $1 \mathrm{~mm}-10$ meter hole is shown in figures 2 (a) to 2 (d). 

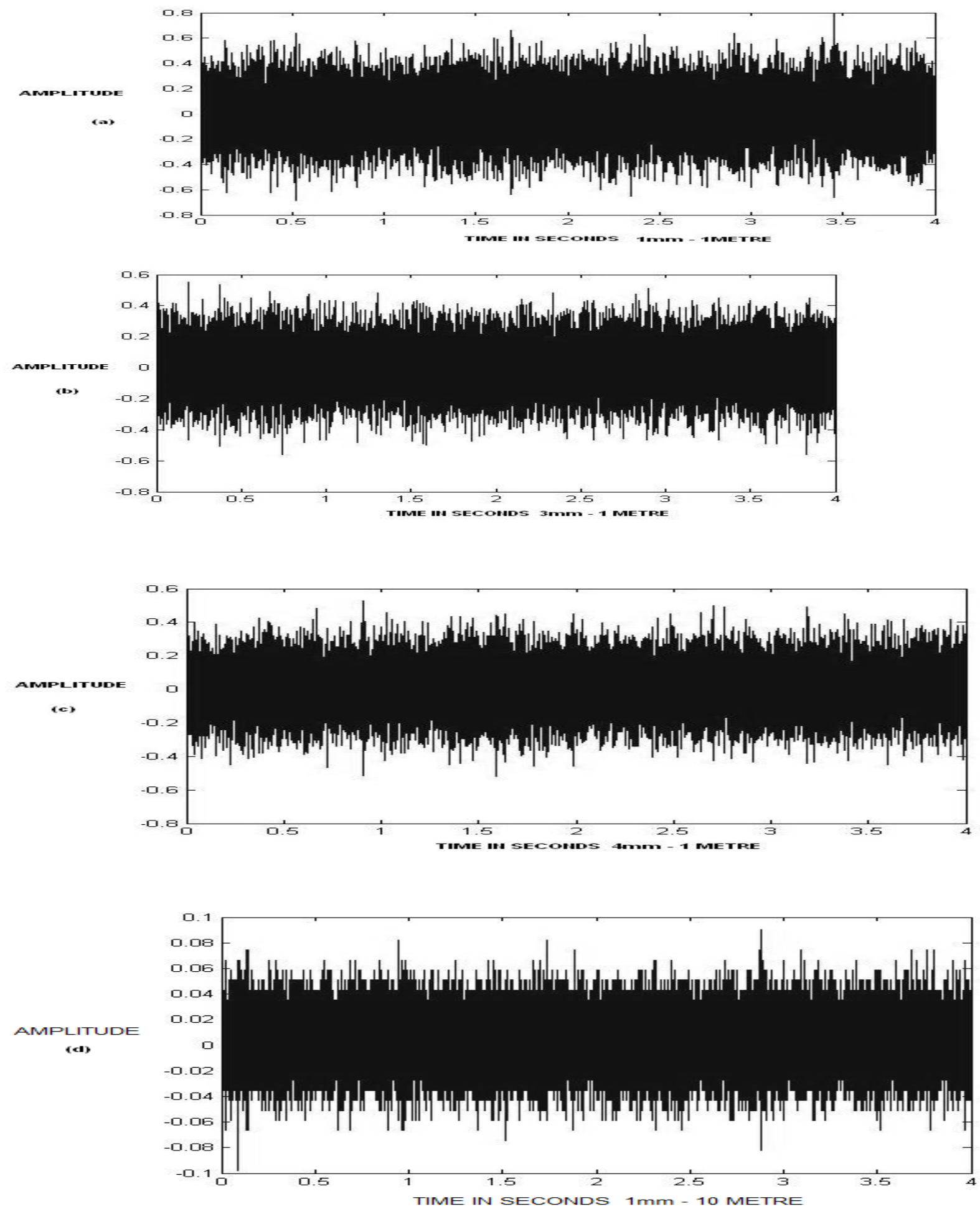

Fig.2. The recorded signal for (a) $1 \mathrm{~mm}-1$ meter hole (b) $3 \mathrm{~mm}-1$ meter hole (c) $4 \mathrm{~mm}$ - 1 meter hole (d) $1 \mathrm{~mm}-10$ meter hole

Similarly the signals for $3 \mathrm{~mm}-1 \mathrm{metre}, 3 \mathrm{~mm}-2 \mathrm{metre}$, and $3 \mathrm{~mm}$ - 5metre and 3mm- 10metre and $4 \mathrm{~mm}-1$ metre, $4 \mathrm{~mm}-2 \mathrm{metre}$, $4 \mathrm{~mm}-5 \mathrm{metre}$ and $4 \mathrm{~mm}-10 \mathrm{metre}$ data is recorded and stored. The acoustic signal from the fabricated setup is filtered using a Gaussian filter. Figure 3 shows the original signal and the filtered signal. After the signal is filtered the features such as
Energy, entropy of the signal, and statistical features such as mean, minimum values of the signal are extracted. These features are tabulated in Table1. The extracted features are then fed as input to the neural network for classification. 



Figure 3. Original and filtered acoustic signal for $1 \mathrm{~mm}$ hole -1 meter distance

Table1. Features extracted for different acoustic leakage signals

\begin{tabular}{|l|c|c|c|c|}
\hline \multicolumn{1}{|c|}{ Holes- dist } & Energy & Entropy & Minimum & Mean \\
\hline 1mm-1meter & 0.32 & 13.12 & $-7.59 E-07$ & 0.52 \\
\hline 1mm-2meter & 0.31 & 12.60 & $-5.43 E-06$ & 0.49 \\
\hline 1mm-5meter & 0.29 & 12.60 & $-4.1 E-06$ & 0.48 \\
\hline 1mm-10meter & 0.29 & 11.07 & $-6.84 E-06$ & 0.43 \\
\hline 3mm-1meter & 0.27 & 12.85 & $-\mathbf{0 . 0 0 0 4 5 2}$ & 0.49 \\
\hline 3mm-2meter & 0.26 & 12.50 & $2.137 \mathrm{E}-06$ & 0.48 \\
\hline 3mm-5meter & 0.25 & 13.07 & $-6.85 E-06$ & 0.51 \\
\hline 3mm-10meter & 0.22 & 12.12 & $-3.42 E-06$ & 0.47 \\
\hline 4mm-1meter & 0.20 & 13.02 & 0.0005748 & 0.50 \\
\hline 4mm-2meter & 0.19 & 13.00 & $3.636 E-07$ & 0.50 \\
\hline 4mm-5meter & 0.19 & 13.16 & $-2.77 E-06$ & 0.52 \\
\hline 4mm-10meter & 0.18 & 12.51 & $8.409 E-06$ & 0.49 \\
\hline
\end{tabular}

In order to train the network, the feed-forward back propagation algorithm is used. The features extracted is taken as input to the neural network and trained. The network architecture is shown in figure 4. It is found that the efficiency of classification and the training time were less when tan-sigmoid function was used for the first layer and purelin for the second layer. Hence the activation functions are selected accordingly Many ANN models, having hidden layer neurons up to 100 , were investigated for determining how changes in the number of neurons in hidden layer contribute to the overall performance of the classification system. . It is noted that learning of the training set does not necessarily guarantee successful diagnostic classification of the test set. The results of neural network models trained with quasi-Newton back propagation algorithm, which was found to be the best training algorithm, is summarized in Table 2 .

Average value of the classification efficiencies obtained on simulation is shown in the Table 2. $\eta_{1}$ is the average efficiency when training set is presented to the trained ANN and $\eta_{2}$ is that when the validation test set is presented.

Table 2. The performance of learning model

\begin{tabular}{|c|c|c|c|c|c|}
\hline $\begin{array}{c}\text { Model } \\
\text { No }\end{array}$ & $\begin{array}{c}\text { ANN } \\
\text { Architecture }\end{array}$ & $\begin{array}{c}\text { No of } \\
\text { Epochs }\end{array}$ & $\begin{array}{c}\text { Training } \\
\text { Time }\end{array}$ & $\begin{array}{c}\text { Efficiency } \\
\eta_{1}\end{array}$ & $\begin{array}{c}\text { Efficiency } \\
\eta_{2}\end{array}$ \\
\hline 1 & $4-5-12$ & 152 & 1.78 & 100 & 88 \\
\hline 2 & $4-10-12$ & 31 & 0.93 & 100 & 93 \\
\hline 3 & $4-35-12$ & 49 & 4.50 & 100 & 82.6 \\
\hline 4 & $4-50-12$ & 212 & 50.46 & 100 & 91.4 \\
\hline 5 & $4-100-12$ & 78 & 83.80 & 100 & 90 \\
\hline
\end{tabular}

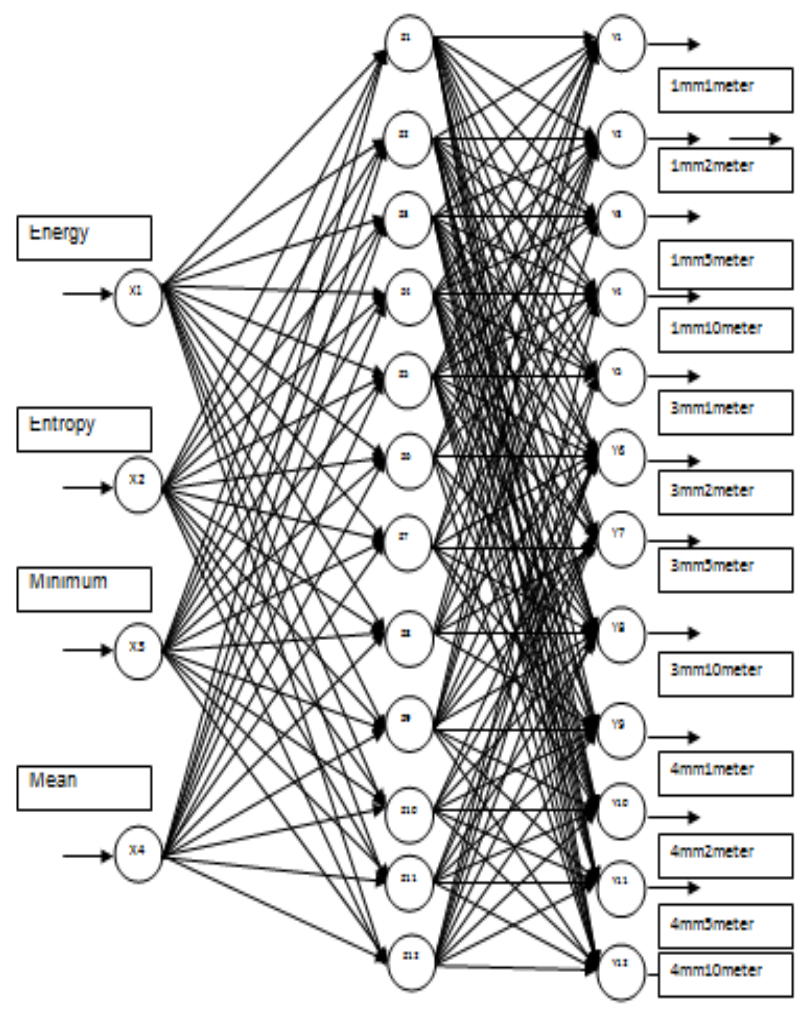

Figure 4. Network Architecture

It was noticed that the best performance was obtained for the training set and validation test set with those models whose hidden layer had 10 neurons. Thus the optimum number of neurons required in the hidden layer is 10 , and hence we have chosen the ANN configuration 4-10-12 which is shown in figure 4. 
Table 3. The performance estimators for features classified using BPN

\begin{tabular}{|l|c|}
\hline \multicolumn{1}{|c|}{ Indices } & BPN \\
\hline Overall Accuracy & $93 \%$ \\
\hline Sensitivity & $92 \%$ \\
\hline Specificity & $94 \%$ \\
\hline
\end{tabular}

The performance estimators for features classified using BPN method is shown in Table 3. The performance estimators include the sensitivity, specificity, and Accuracy. Using all the features obtained from the recorded signal the sensitivity and specificity were found to be $92 \%$ and $94 \%$ respectively.

\section{CONCLUSION}

In this study the features of the acquired signal from the prototype is extracted, filtered using a Gaussian filter and trained in a neural network and the following observations were done. When there is a leak the energy level of the acquired signal is higher compared to the background noise signals. From this we infer that the leak is present nearby. As the size of the leak increases the energy level of the signal decreases which shows that the leakage is present at a limited distance. Our future work is to find the exact location of the tube leakage. Some of the benefits that can be obtained by implementing this technique are increased personnel safety, early warning small tube leak can prevent extensive damage, Increased availability and increase in plant efficiency, reduce repair time and safeguard your investment, planned and orderly shutdown by boiler, increased operating profits and saving of fuel due to planned and scheduled shutdown. In view of the foregoing we can find out the leakage using intelligent technique.

\section{REFERENCES}

[1] Bjorn Widarsson, Erik Dotzauer, "Bayesian network-based early-warning for leakage in recovery boilers," Applied thermal Engg 2008, vol.28, pp. 754-760.

[2] Er Ashutosh Kumar Gupta , Lamar Stonecypher , "Boiler Tube Leakage detection by Acoustic Instrument in Thermal Power Plant", http://www.brighthubengineering.com, 2010
[3] Buckner G.D., Marziale M.L., "Early steam leak detection can help limit boiler damage", avert explosion, pulp pap, 66(9),1992, pp.167-170.

[4] B.Studdard, P.Arrington, "Early boiler leak detection cuts costs," Power Engg. 97 (9), 1993, pp. 25-27.

[5] Cinar, C. Undey, "Statistical Process and controller performance monitoring," Proceedings of the American Control Conference, 1999, pp. 2625-2639.

[6] Yongxing Lv, Qiang Feng, "Study on Acoustic Catheter of Boiler Tube Leakage Monitoring Systems," Physics Procedia 2012, Vol.33, pp. 641-646.

[7] Yoon S, MacGregor, J.F, "Statistical and casual model based approaches to fault detection and isolation," AIChE J 2000 , vol. 46, pp. 1813-1824.

[8] Xi Sun, Horacio J. Marquez, Tongwen Chen, "An improved PCA method with application to boiler leak detection," ISA Transaction 2005, vol. 44, pp. 379-397.

[9] Sujatha CM, MaheshV, Swaminathan Ramakrishnan, Comparison of Two ANN Methods for Classification of Spirometer Data. Measurement Science Review. 2008; 8(3): $53-57$.

[10] Najumnissa, D and Rangaswamy, "Comparison of Intelligent computing techniques for classification of Clinical EEG signals", European Journal of Biomedical Informatics, volume 9, (2), 2013.

[11] Ubeyli ED, Guler I, "Adaptive neuro-fuzzy inference systems for analysis of internal carotid arterial Doppler signals”, Comput. Biol. Med. 2005; 35: 687-702.

[12] Jang JSR, ANFIS: adaptive network based fuzzy Inference system, IEEE Trans. Syst., Man Cybern. 1993; 23, (3): 665-683.

[13] Cvejic, N., D. Bull and N. Canagarajah, "Region based multimodal image fusion using ICA bases", IEEE Sensors Journal, 2007. 7: 743-751.

[14] Lindasalwa Muda, Mumtaj Begam, Elamvazuthi. I., "Voice Recognition Algorithms using Mel Frequency Cepstral Coefficient (MFCC) and Dynamic Time Warping (DTW) Techniques", Journal of Computing, Vol. 2, (3), 2010 